(11.4\%) subjects with 4 being exposed to higher dust levels. There is a statistically significant relationship with duration of work, type of work and the level of dust exposure with the clinical features $(\mathrm{p}<0.001)$.

Conclusion There is a decline in the lung function which is related to the duration of work and amount of dust exposure. It mainly shows a restrictive pattern of impairment.

\section{P1-450 IMPROVEMENTS IN SOCIAL COHESION: DO THEY MATTER FOR PHYSICAL ACTIVITY}

doi:10.1136/jech.2011.142976g.40

${ }^{1} \mathrm{~B}$ Jongeneel, ${ }^{*}{ }^{1} \mathrm{~W}$ Busschers, ${ }^{1,2} \mathrm{M}$ Droomers, ${ }^{1} \mathrm{~K}$ Stronks, ${ }^{1} \mathrm{~A}$ Kunst. ${ }^{1}$ Academic Medical Center, University of Amsterdam, Amsterdam, The Netherlands; ${ }^{2}$ Centre for Prevention and Health Services Research, National Institute for Public Health and the Environment, Bilthoven, The Netherlands

Introduction Much research exists on the relationship between environmental factors and physical activity (PA). However, few studies have investigated the role of social cohesion in neighbourhoods. The aim of this study was to assess the extent to which social cohesion and changes over time in the levels of social cohesion were associated with PA.

Methods We used data from the survey WoonOnderzoek Nederland 2006 and 2009, using two samples of 57092 Dutch adults. Using multi-level Hurdle Negative Binomial models, we assessed if the odds for being physically inactive and the mean hours of PA among people who are physically active were related to levels of social cohesion at neighbourhood level, and changes in social cohesion between 2006 and 2009. Next, we examined if these associations varied according to sex, age, and economic activity.

Results In areas with higher levels of social cohesion compared to areas with less social cohesion, more people were physically active. In addition, generally social cohesion was not associated with the amount of PA. Improvements in social cohesion between 2006 and 2009 were not associated with the odds of being inactive in the general population and with the amount of PA among the people being physically active.

Conclusion Social cohesion is related to physical (in) activity. However, improvements in social cohesion were not strongly related to physical (in) activity. This raises question on the causality of the relationships that are observed in cross-sectional analyses. Enhancing social cohesion in neighbourhoods may have limited influence on population levels of physical activity.

\section{P1-451 INFANT MORTALITY IN SWITZERLAND}

doi:10.1136/jech.2011.142976g.41

C Junker, ${ }^{*}$ S Berrut. Federal Statistical Office, Neuchâtel, Switzerland

Infant mortality rates in Switzerland had been among the lowest in Europe. This is not longer the case. Swiss rates are now in the medium range. This paper examines reasons why. Data from 1900 to 2009 is based on the birth database hold at the Swiss Federal Statistical Office (FSO). Variables on weight, length, number of siblings, ages of mother and father are taken into account, as well as causes of death. Gestational age was only recently introduced into the data registration. In 1900 the infant mortality rate in Switzerland was 150 per 1000 life births, in 19906.8 and in 20094.3 per 1000. In the last 20 years, the infant mortality rate dropped by about a third. A strong decline is primarily observed among children aged 28 days up to 1 year. In children aged 1 to 27 days, the mortality rate has halved over the same period. A growing mortality rate, however, is seen in infants in the first $24 \mathrm{~h}$ after birth. Mostly affected are extremely premature births, which are due to their immaturity at high risk. During the same time, numbers of twins, numbers of low birth weight infants and age of mothers had increased considerably. The slowing, if not stagnant decline in infant mortality in Switzerland in recent years is explained by an increase in high risk deliveries. Infants in Switzerland die, if anything, more and more in the first days of life, even in the first hours after birth.

\section{P1-452 BREASTFEEDING PRACTICES IN INDIA: A SURVIVAL ANALYSIS}

doi:10.1136/jech.2011.142976g.42

\section{S Kansal.* Banaras Hindu University, Varanasi, India}

Introduction The single most cost effective intervention to reduce infant mortality in developing countries would be by promoting exclusive breastfeeding. Breastfeeding should be initiated immediately after child birth and should be continued exclusively up to a maximum of 6 months. In India where a majority of the population has a low income and poor education, the need for breastfeeding represents the effective way of giving child a fair chance of survival and good health. The objective of the present study was to describe the association between exclusive breastfeeding and socio-economic demographic \& cultural variables.

Methods The data for the study was taken from nationwide District Level Health Survey- 3 (DLHS-3) conducted in 2007-2008. The statistical tests used were Kaplan-Meier survival curves \& Cox proportional hazard model.

Results The mean duration of exclusive breastfeeding in India was found 3.31 months (95\% CI 3.08 to 3.15 ) while it was found almost 4.15 months (4.03 to 4.28 ) in low infant mortality states in southern India compared to 1.5 months (1.45 to 1.54 ) in high infant mortality states in northern India. The analysis showed that no maternal education $(p<0.001)$, being an unemployed mother $(p<0.001)$, and Muslims $(p<0.001)$ were important associations of early cessation of exclusive breastfeeding.

Conclusion The study showed that survival status of the child had a significant impact on the duration of exclusive breastfeeding. Also it was found that the time of initiation of breastfeeding after birth was an important determinant for total duration of exclusive breastfeeding.

\section{P1-453 TRENDS OF VASCULAR SURGERY IN SCOTLAND 1991-2007}

doi:10.1136/jech.2011.142976g.43

${ }^{1,2}{ }^{K}$ Karim, ${ }^{*}{ }^{2} \mathrm{~J}$ Lewsey, ${ }^{2} \mathrm{~S}$ Inglis. ${ }^{1}$ Iraqi Centre for Heart Diseases, Baghdad, Iraq; ${ }^{2}$ University of Glasgow, Glasgow, UK

Objective To understand trends of vascular interventions and changes in provision of vascular surgery.

Design A retrospective, descriptive, population-based study using hospital discharge data.

Setting Scotland 1991-2007.

Chief outcome measures Time trends in patient demographics and age and sex-specific population rates of these procedures.

Results In Scotland, between 1991 and 2007, a total of 153117 vascular procedures of interest were performed. The proportion of men who underwent these procedures was higher than the proportion of women. The mean age of individuals who underwent amputation and lumbar sympathectomy decreased significantly over the period under review $(p<0.001)$. In contrast, the mean age of individuals who underwent diagnostic endovascular procedures and therapeutic endovascular procedures increased significantly $(p<0.001)$. However, the mean age of individuals who underwent 